In: Ansgar Beckermann

Aufsätze, Band 3

Bielefeld: Universitätsbibliothek Bielefeld 2021

DOI: https://doi.org/10.4119/unibi/2950621) 


\title{
Fähigkeitsbasierte Freiheitstheorien und das Problem des Determinismus*
}

\author{
zusammen mit Romy Jaster
}

\section{Einleitung}

Eine Person ist in ihrem Handeln frei, wenn sie tun kann, was sie tun will. Aber wann ist sie in ihrem Wollen frei? Die Antwort auf diese Frage scheint schwierig; aber im Laufe der Zeit ist doch immer wieder die Auffassung vertreten worden, dass eine Person genau dann in ihrem Wollen frei ist, wenn sie über bestimmte Fähigkeiten verfügt, wenn sie insbesondere etwas anders machen kann, als sie es tatsächlich tut. ${ }^{1}$ So schreibt etwa John Locke im 21. Kapitel des zweiten Buches seines Essay concerning Human Understanding:

There being in us a great many uneasinesses always solliciting, and ready to determine the will, it is natural, as I have said, that the greatest, and most pressing should determine the will to the next action; and so it does for the most part, but not always. For the mind having in most cases, as is evident in Experience, a power to suspend the execution and satisfaction of any of its desires, and so all, one after another, is at liberty to consider the objects of them; examine them on all sides, and weigh them with others. In this lies the liberty Man has; $[\ldots]$ we have a power to suspend the prosecution of this or that desire, as every one daily may Experiment in himself. This seems to me the source of all liberty; in this seems to consist that, which is (as I think improperly) call'd Free will. For during this suspension of any desire, before the will be determined to action, and the action (which follows that determination) done, we have opportunity to examine, view, and judge, of the good or evil of what we are going to do; and when, upon due Examination, we have judg'd, we have done our duty, all that we can, or ought to do, in pursuit of our happiness; and 'tis not a fault, but a perfection of our nature to desire, will, and act

* Zuerst erschienen in: Zeitschrift für philosophische Forschung 72 (2018), S. 317-342 (Vittorio Klostermann Verlag).

1 Wenn man so will, findet diese Auffassung einen prominenten Ausdruck schon im Prinzip alternativer Möglichkeiten, dem zufolge eine Person in ihrem Wollen nur dann frei ist, wenn sie anders hätte handeln bzw. entscheiden können. 
according to the last result of a fair Examination. (Locke 1975, 263f. - Hervorh. im Original)

Für Locke beruht Willensfreiheit ${ }^{2}$ also darauf, dass wir - zumindest in vielen Fällen - die Fähigkeit besitzen, vor einer Entscheidung innezuhalten und zu überlegen, was wir in der gegebenen Situation tun sollten - was moralisch richtig wäre und was unseren wohlverstandenen Eigeninteressen am meisten nützen würde. Doch das reicht noch nicht aus. Willensfreiheit setzt auch voraus, dass wir dem Ergebnis der eigenen Überlegung gemäß entscheiden (und dann entsprechend handeln) können. In seinem Wollen frei ist ein Akteur nach Locke also genau dann, wenn er über zwei Fähigkeiten verfügt - die Fähigkeit, vor dem Handeln innezuhalten und zu überlegen, und die Fähigkeit, dem Ergebnis dieser Überlegung gemäß zu entscheiden und zu handeln.

Damit formuliert Locke eindrucksvoll eine fähigkeitsbasierte Theorie der Willensfreiheit. Allerdings steht Locke mit einer solchen Theorie keineswegs allein. Vielmehr kann er sich unter anderem auf eine lange Tradition in der Rechtstheorie berufen. Schon im Römischen Strafrecht findet sich der Grundsatz, dass zwei Gruppen von Menschen grundsätzlich nicht schuldfähig sind - Kinder und psychisch Kranke. ${ }^{3}$ Warum ist das so? Im $\S 20$ unseres Strafgesetzbuches, in dem es um die Schuldunfähigkeit psychisch Kranker geht, findet sich eine Antwort: ${ }^{4}$

Ohne Schuld handelt, wer bei Begehung der Tat wegen einer krankhaften seelischen Störung, wegen einer tiefgreifenden Bewußtseinsstörung oder wegen Schwachsinns oder einer schweren anderen seelischen Abartigkeit unfähig ist, das Unrecht der Tat einzusehen oder nach dieser Einsicht zu handeln. (StGB $\S 20$ - unsere Hervorh.)

Auch unserem Strafrecht zufolge beruht Schuldfähigkeit also offenbar auf dem Vorhandensein zweier Fähigkeiten - der Fähigkeit, das Unrecht der Tat einzusehen, und der Fähigkeit, dieser Einsicht gemäß zu handeln.

2 Locke hält den Ausdruck „Willensfreiheit“ für unangemessen, da es in seinen Augen nicht darum geht, ob unser Wille frei ist (das ist nach Locke gar nicht möglich), sondern ob wir in unserem Wollen frei sind. Doch das spielt im Folgenden keine Rolle; wir verwenden deshalb die allgemein übliche Terminologie.

3 Zu weiteren Einzelheiten vgl. Beckermann 2016, $9 f$.

4 In $\S 19$ StGB wird zunächst festgestellt, dass Kinder unter 14 Jahren schuldunfähig sind. 
In den letzten Jahren hat auch Geert Keil eine fähigkeitsbasierte Theorie der Willensfreiheit entwickelt, die auf den ersten Blick eine große Ähnlichkeit zu der Lockes aufweist:

Positiv wurde Willensfreiheit oben [...] als das komplexe Vermögen erläutert, praktische Überlegungen anzustellen, bestehende eigene Wünsche zu prüfen und gegebenenfalls zu suspendieren und das Ergebnis dieses Abwägungsprozesses handlungswirksam werden zu lassen. (Keil 2013, 146)

Bei näherem Hinsehen ist in Keils Theorie insbesondere entscheidend, dass der Akteur zu dem Zeitpunkt, an dem er am Ende seiner Überlegungen zu einer Entscheidung kommt, immer noch die Fähigkeit besitzt, weiter zu überlegen (Keil 2009, ((60))). Allerdings schreibt Keil auch:

Ich fasse das Weiterüberlegen nicht als eine separate Fähigkeit auf, die zur Fähigkeit, praktische Überlegungen anzustellen, noch hinzukäme. Weiterzuüberlegen ist eine weitere gleichartige Phase der Tätigkeit des Überlegens. (Keil 2013, 224 - Hervorh. im Original)

Die Unterschiede zwischen verschiedenen fähigkeitsbasierten Freiheitstheorien sind für die folgenden Überlegungen ohne größere Bedeutung. Denn uns geht es hier vor allem um die Frage, ob ein Akteur die für Freiheit und Verantwortlichkeit entscheidenden Fähigkeiten auch besitzen kann, wenn der Determinismus wahr ist. Auch diese Frage wird von Keil aufgeworfen:

[...] Beckermann und Tugendhat rücken beispielsweise das Lockesche Suspensionsvermögen in das Zentrum ihrer Freiheitsauffassungen. Allerdings sind sie der Auffassung, dass dieses Vermögen auch in einer deterministischen Welt existieren und ausgeübt werden kann. Dagegen argumentieren Libertarier, dass Willensfreiheit, die ich als die Fähigkeit der überlegten hindernisüberwindenden Willensbildung und -umsetzung verstehe, sowohl durch den pathologischen Verlust von Einsichts-und Steuerungsfähigkeiten als auch durch den Determinismus tangiert wird. (Keil 2013, 178 - Hervorh. im Original)

Keil bezweifelt, dass Lockes Suspensionsvermögen tatsächlich mit einem strengen Determinismus vereinbar ist, dem zufolge jeder beliebige Weltzustand gemeinsam mit den Naturgesetzen jeden späteren Weltzustand festlegt. ${ }^{5}$ Für Keil gilt: Wenn der Überlegens- und Entscheidungsprozess ein in diesem Sinne deterministischer Prozess wäre, besäße kein Akteur je die Fähigkeit, innezuhalten und seine Entscheidung zu überprüfen. Wäre „das Überlegen in Wirklichkeit ein naturgesetzlicher Mechanismus mit determiniertem Ausgang [, dann] wäre aus inkompatibilistischer Sicht das Vermögen des Innehaltens und Prüfens eine bloße Illusion, denn alternative Über-

5 Wir folgen hier der Standarddefinition; vgl. Hoefer 2016. 
legungswege wären tatsächlich naturgesetzlich verschlossen“ (ebd., 59). Gerade die für Freiheit entscheidenden Fähigkeiten kann man also laut Keil nur besitzen, wenn der Determinismus falsch ist.

Um genau diese Frage soll es im Folgenden gehen: Kann ein Mensch die für Freiheit entscheidenden Fähigkeiten auch dann besitzen, wenn der Weltverlauf vollständig determiniert ist? Oder hat Keil Recht, dass Freiheit auch in diesem Sinn mit Determiniertheit unvereinbar ist? Die Frage Keils stellt sich für jede fähigkeitsbasierte Freiheitstheorie unabhängig davon, welche Fähigkeiten jeweils für Freiheit als konstitutiv angesehen werden. Wir werden uns im Folgenden aber hauptsächlich auf die beiden Lockeschen Fähigkeiten konzentrieren, da sich an ihnen wie uns scheint die grundsätzlichen Probleme und Lösungsmöglichkeiten sehr gut exemplarisch erläutern lassen.

Unsere These wird sein, dass Akteure auch in einer deterministischen Welt über die freiheitsrelevanten Fähigkeiten verfügen können. Unser Argument hat vier Schritte. Im nächsten Abschnitt argumentieren wir dafür, dass eine Fähigkeitstheorie bestimmte Anforderungen erfüllen muss, die sich im Zusammenhang mit freiheitsrelevanten Fähigkeiten als relevant erweisen werden, und stellen exemplarisch eine Theorie vor, die diesen Anforderungen gerecht wird. Im dritten Abschnitt unterscheiden wir sorgfältig zwischen unterschiedlichen Arten von Fähigkeiten. In Abschnitt 4 zeigen wir, dass nur eine ganz bestimmte Sorte von Fähigkeiten mit dem Determinismus unvereinbar ist und diagnostizieren den entscheidenden Disput zwischen Kompatibilisten und Inkompatibilisten. In Abschnitt 5, schließlich, argumentieren wir dafür, dass wir gute Gründe für die Annahme haben, dass diese determinismusinkompatible Sorte von Fähigkeiten nicht diejenige ist, die für Willensfreiheit relevant ist.

\section{Fähigkeiten}

Um sich der Frage nach der Vereinbarkeit von freiheitsrelevanten Fähigkeiten und Determinismus anzunähern, empfiehlt es sich, zunächst einen Schritt zurückzutreten und zu fragen, was es mit Fähigkeiten ganz allgemein auf sich hat. Eine sehr einflussreiche Antwort hat Moore mit seiner sogenannten konditionalen Analyse gegeben.

If by saying that we could have done, what we did not do, we often mean merely that we should have done it, if we had chose to do it, by saying that we could have chosen to do it, we may mean merely that we should have so chosen, if we had chosen to make the choice. (Moore 1996, 113f. - Hervorh. im Original) 
Der konditionalen Analyse zufolge hat ein Akteur genau dann die Fähigkeit, $\varphi$ zu tun, wenn ein bestimmtes kontrafaktisches Konditional wahr ist: Wenn sich der Akteur entscheiden würde, $\varphi$ zu tun, würde er $\varphi$ tun. In der Semantik möglicher Welten können wir sagen, dass ein Akteur genau dann die Fähigkeit hat, $\varphi$ zu tun, wenn er in den nächsten Welten, in denen er sich entscheidet, $\varphi$ zu tun, auch tatsächlich $\varphi$ tut. ${ }^{6}$ Der Kerngedanke der konditionalen Analyse besteht demnach darin, eine modale Verbindung zwischen der Entscheidung des Akteurs und der erfolgreichen Ausführung der entsprechenden Handlung zu postulieren.

Die konditionale Analyse spielt Kompatibilisten in die Karten. Denn, wie leicht zu sehen ist, kann die konditionale Analyse auch dann erfüllt sein, wenn der Akteur sich nicht dazu entscheidet, $\varphi$ zu tun, und es deshalb auch nicht tut. Alles, was zählt, ist, dass er es tun würde, wenn er sich dazu entscheiden würde. Diese Auffassung von Fähigkeitszuschreibungen erlaubt Moore seinen berühmten Zug gegen den Inkompatibilismus: Selbst wenn determiniert ist, dass der Akteur eine bestimmte Handlung nicht ausführen wird, kann er dennoch die Fähigkeit besitzen, die Handlung auszuführen. Die Fähigkeit, anders zu handeln, wäre demnach mit dem Determinismus vereinbar.

Moores Position gilt heute als unhaltbar. Die Fähigkeitstheorie, auf der sie beruht, hat sich inzwischen als falsch erwiesen. Denn obwohl Moore richtig sieht, dass man, um eine Fähigkeit zu haben, sie weder ausüben, noch sich entscheiden muss, sie auszuüben, übersieht er, dass es zumindest möglich sein muss, die entsprechende Entscheidung zu treffen.

Ein Komapatient kann offenkundig nicht aus dem Bett aufstehen. ${ }^{7}$ Aber der Grund für seine fehlende Fähigkeit ist nicht, dass das von Moore postulierte Konditional in dem Fall nicht erfüllt wäre. Ganz im Gegenteil; in den nächsten Welten, in denen der Komapatient sich entscheiden würde aufzustehen, würde er es wohl tun - schließlich wäre er in diesen Welten nicht im Koma. Dass er die Fähigkeit dennoch nicht hat, scheint daran zu liegen, dass sich der Komapatient angesichts seines Komas gar nicht entscheiden kann aufzustehen. Die konditionale Analyse liefert hier das falsche Ergebnis.

Nun kann ein Komapatient sich nicht nur nicht entscheiden, aus dem Bett aufzustehen, sondern überhaupt keine Entscheidung treffen. Das ist

6 Das ist nur ungefähr richtig. Wir ignorieren der Einfachheit halber die Probleme, die sich durch Trivialität und mit der so genannten „limit assumption“ ergeben.

7 Van Inwagen 1983, 119. 
aber nicht entscheidend. Der Fall eines Phobikers, der keine Spinnen anfassen kann, ${ }^{8}$ ist in den relevanten Hinsichten analog. Wenn der Phobiker sich entscheiden würde, die Spinne zu berühren, würde er es tun. Sein Problem ist ja nicht motorischer Art. Vielmehr kann der Phobiker sich gar nicht zur Entscheidung durchringen. Auch hier ist also das kontrafaktische Konditional erfüllt, obgleich dem Akteur die Fähigkeit fehlt.

Was läuft bei der konditionalen Analyse schief? Das Problem hat mit der kontrafaktischen Struktur der Analyse zu tun und sitzt insofern ziemlich tief. Das Konditional ist immer dann wahr, wenn der Akteur in den nächsten Welten, in denen er sich entscheidet, $\varphi$ zu tun, auch tatsächlich $\varphi$ tut. Aber in manchen Fällen kann der Akteur sich gar nicht erst entscheiden, die Handlung auszuführen. Um die nächsten Entscheidungswelten zu erreichen, müssten wir in solchen Fällen also hinter die Welten zurückgehen, in denen die Bedingungen vorliegen, die den Akteur tatsächlich an der Entscheidung hindern. Im Komafall etwa müssten wir in Welten vorrücken, in denen der Akteur gar nicht im Koma liegt. In solchen Welten spricht dann aber klarerweise nichts mehr gegen eine erfolgreiche Ausführung. Das Konditional ist erfüllt, aber der Akteur hat dennoch nicht die Fähigkeit. Nennen wir Gegenbeispiele dieser Art „Fälle der verhinderten Entscheidung". ${ }^{9}$ Sie sind, historisch gesprochen, der Hauptgrund dafür, dass die konditionale Analyse heute als unhaltbar gilt. ${ }^{10}$

Dennoch hat Moore einige ganz grundlegende Dinge richtig gesehen. Erstens: Fähigkeiten sind modale Eigenschaften. Um die Fähigkeit zu haben, etwas zu tun, muss ein Akteur sich weder zur Ausübung entscheiden noch die Handlung tatsächlich ausführen. Entscheidung und Ausführung müssen ihm nur in einem bestimmten Sinn möglich sein. Und zweitens: Fähigkeiten haben etwas mit der modalen Verbindung zwischen der Entscheidung des Akteurs und der erfolgreichen Ausführung der entsprechenden Handlung zu tun.

Die offene Frage ist, wie man diesen Punkten im Rahmen einer Fähigkeitstheorie Rechnung tragen sollte. Hier ein Vorschlag:

8 Lehrer 1969.

9 Neben van Inwagen und Lehrer haben auch Chisholm $(1966,1976)$ und Whittle (2010) das Problem gesehen. Auch bei Moore selbst ist es bereits angerissen (Moore 1966, Kap. 6).

10 Es gibt allerdings noch weitere wichtige Gründe: Die konditionale Analyse hat Probleme mit der Maskierbarkeit und Graden von Fähigkeiten (vgl. Manley \& Wasserman 2008). Darüber hinaus kann sie auch die Unterscheidung zwischen generellen und speziellen Fähigkeiten nicht einfangen (Jaster 2016). 
ERFOLG. Ein Akteur hat genau dann die Fähigkeit, $\varphi$ zu tun, wenn er in einer hinreichenden Anzahl von relevanten möglichen Situationen, in denen er sich entscheidet, $\varphi$ zu tun (bzw. wie wir im Folgenden sagen werden: in denen er die Intention ausbildet, $\varphi$ zu tun), auch tatsächlich erfolgreich $\varphi$ tut.

Nennen wir dies die „Erfolgstheorie von Fähigkeiten“. ${ }^{11}$ Die Erfolgstheorie wird im Detail in Jaster (2016) entwickelt und ausführlich verteidigt. Ähnliche Ansätze sind in den letzten Jahren von Greco (2009), Maier (2013), Vihvelin $(2004,2013)$ und - in der Dispositionendebatte - von Manley und Wasserman (2008) formuliert worden. Die Unterschiede zwischen diesen Theorien sind an dieser Stelle nicht entscheidend. Wichtig ist lediglich die Feststellung, dass die Erfolgstheorie zwei grundlegende Einsichten kombiniert, denen jede Fähigkeitstheorie Rechnung tragen muss und uns daher das Handwerkszeug liefert, das für die weitere Diskussion entscheidend sein wird.

Erste grundlegende Einsicht: Wir haben Fähigkeiten immer angesichts bestimmter Tatsachen. Wie Angelika Kratzer schreibt, kann man mit einem Satz wie beispielsweise „Ich kann nicht Posaune spielen“ ganz unterschiedliche Aussagen machen.

I may mean that I don't know how to play the trombone. I am sure that there is something in a person's mind which becomes different when he or she starts learning how to play the trombone. A programme is filled in. And it is in view of this programme that it may be possible that I play the trombone. Or suppose that I suffer from asthma. I can hardly breathe. In view of my physical condition I am not able to play the trombone, although I know how to do it. I may express this by uttering [,I cannot play the trombone"]. Or else imagine that I am travelling by sea. The ship sinks and so does my trombone. I manage to get to a lonely island and sadly mumble [,I cannot play the trombone"]. I could play the trombone in view of my head and my lungs, but the trombone is out of reach. (Kratzer 1981, 54)

Kratzer bedient sich in dieser Passage der Konstruktion „can in view of", um deutlich zu machen, dass Akteure Fähigkeiten immer nur angesichts bestimmter Gegebenheiten in der Welt haben. Und welche Gegebenheiten uns interessieren, variiert je nach Situation. ${ }^{12}$

${ }^{11}$ In Jaster 2016 verwendet Romy Jaster die Bezeichnung „The Success View of Abilities".

12 Ähnlich äußert sich David Lewis in (Lewis 1976). Wie Kratzer selbst (Kratzer 1977, 1981) ist auch Lewis der Auffassung, dass Fähigkeitszuschreibungen Möglichkeitsaussagen sind. "S kann $\varphi$ tun" drückt demnach aus, dass es, in 
Entsprechend variieren die relevanten möglichen Situationen, die in der Erfolgstheorie eine entscheidende Rolle spielen. Relevant sind die Situationen, in denen die Gegebenheiten bestehen, die uns in einem Kontext interessieren. Fähigkeitszuschreibungen funktionieren hier ganz analog zu eingeschränkten Möglichkeits- oder Notwendigkeitsaussagen. Wie Barbara Vetter ausführt, kann eine Möglichkeitsaussage wie „Hydrangeas can grow here" je nach Kontext ebenfalls ganz unterschiedliche Propositionen ausdrücken.

[W] hen a botanist says, in a foreign country with unfamiliar vegetation, 'Hydrangeas can grow here', she says not just that in some metaphysically possible worlds there are hydrangeas growing on this soil. She speaks only about worlds where the biology of hydrangeas, the geology and the climate of the country are as they are in actuality. When a detective says, 'John [cannot] be the murderer', she speaks about a different set of worlds: those that are compatible with everything she knows. In each case the conversational background selects a set of propositions - about biology and geology, or about anything that the detective knows. The modal base is the set of worlds in which those propositions are true. (Vetter 2015, 68)

In allen genannten Verwendungen der Möglichkeitsaussage „Hydrangeas can grow here" sind die möglichen Welten auf eine Teilmenge eingeschränkt; die Welten, in denen die Fakten bestehen, die uns im jeweiligen Kontext interessieren - die Tatsachen, angesichts derer die Sache möglich ist. Etwas Terminologie erweist sich in diesem Zusammenhang als hilfreich. Wir können sagen, dass wir, abhängig vom Kontext, unterschiedliche Tatsachen über die möglichen Welten hinweg festhalten und alle anderen Tatsachen variieren.

Innerhalb der Erfolgstheorie von Fähigkeiten liefern uns die Tatsachen, die wir jeweils festhalten, die relevanten Situationen; die relevanten Situationen sind die Situationen, in denen die Tatsachen bestehen, die uns in einem Kontext interessieren. Alle anderen Tatsachen können variieren.

Natürlich ist es nicht beliebig, welche Tatsachen wir festhalten. Da wir an den Fähigkeiten eines bestimmten Akteurs interessiert sind, halten wir stets einige der intrinsischen Eigenschaften eben jenes Akteurs fest. Darüber hinaus halten wir in der Regel Hintergrundannahmen über die Welt fest. In der Regel halten wir Umstände fest, die wir für normal halten. Und

einem passend eingeschränkten Sinn möglich ist, dass $\mathrm{S} \varphi$ tut; genauer: dass $\mathrm{S}$ in einer der relevanten möglichen Welten $\varphi$ tut. Siehe (Kenny 1976) für eine schlagende Kritik an dieser Auffassung. 
manchmal halten wir beliebig große Mengen von Tatsachen darüber fest, in welcher konkreten Situation sich ein Akteur gerade befindet.

Ein und derselbe Satz der Form „S kann $\varphi$ tun“ drückt entsprechend ganz unterschiedliche Propositionen aus, je nachdem welche Welten in einem Äußerungskontext relevant sind. Auf Kratzers Beispiele angewendet: Der Satz „Ich kann nicht Posaune spielen“ drückt unterschiedliche Propositionen aus, je nachdem, ob wir nur die für das Posaune Spielen relevante Hirnstruktur des Akteurs, sein Asthma oder die Tatsache festhalten, dass er sich nicht in Reichweite einer Posaune befindet.

Die zweite grundlegende Einsicht ist, dass Fähigkeiten zu haben in aller Regel keine Sache eines einmaligen Erfolgs ist, sondern damit zu tun hat, wie hoch die Erfolgsquote der Person über mögliche Situationen hinweg ist - oder wie wir sagen wollen: die modale Erfolgsquote des Akteurs. Eine Fähigkeit hat man laut Erfolgstheorie genau dann, wenn man eine hinreichend hohe modale Erfolgsquote hat. ${ }^{13}$

Welche modale Erfolgsquote hinreichend hoch ist, variiert ebenfalls je nach Kontext. Manchmal genügt es, wenn ein Akteur in einem einzigen unter einer immens hohen Anzahl möglicher Fälle erfolgreich ist. Von einer Läuferin, die den Marathon immer knapp über, aber ein einziges Mal unter 3 Stunden läuft, sagt man in manchen Kontexten korrekterweise, dass sie die 42 Kilometer unter drei Stunden laufen kann. In anderen Kontexten verlangen wir hingegen eine deutlich höhere modale Erfolgsquote. Wenn wir ein Team aus Läuferinnen zusammenstellen wollten, die unter drei Stunden laufen können, wäre unsere Läuferin wahrscheinlich nicht auf der Liste. Hier spielt also offenbar der Kontext die entscheidende Rolle. Die modale Erfolgsquote ist ein wichtiges Element in einer Theorie von Fähigkeiten, weil sie uns Grade von Fähigkeiten liefert. ${ }^{14}$ Dieser Punkt wird aber im weiteren Verlauf keine Rolle mehr spielen.

Wie sich leicht erkennen lässt, hat die Erfolgstheorie gewisse Ähnlichkeiten mit der konditionalen Analyse; darunter die zentrale Gemeinsamkeit, dass Fähigkeiten beiden Theorien zufolge etwas mit der modalen Verbindung zwischen Intention und Ausführung zu tun haben. Doch im Unter-

13 Es ist wichtig, die modale Erfolgsquote von der tatsächlichen Erfolgsquote des Akteurs zu unterscheiden. Wichtig ist, wie hoch die Erfolgsquote über mögliche Situationen ist. Diese Quote kann sich von der tatsächlichen Erfolgsquote - dem „track record“ des Akteurs in der aktualen Welt (Greco 2007, 60f.) stark unterscheiden.

14 Für Dispositionen haben Manley und Wasserman (2008) diesen Punkt herausgestellt. Für Fähigkeiten siehe Jaster (2016). 
schied zur konditionalen Analyse buchstabiert die Erfolgstheorie die relevante Modalverbindung nicht in Form eines kontrafaktischen Konditonals aus.

Dieser Unterschied ist in einer Reihe von Hinsichten von entscheidender Bedeutung. ${ }^{15}$ In unserem Zusammenhang ist vor allem ein Punkt wichtig: Die Erfolgstheorie umgeht das Problem der verhinderten Entscheidung, weil ihr zufolge eine Fähigkeit nur dann vorliegen kann, wenn es dem Akteur tatsächlich möglich ist, sich zu entscheiden bzw. zu intendieren, die Handlung auszuführen.

Nehmen wir zur Veranschaulichung noch einmal den Komafall. Der Komapatient kann nicht aus dem Bett aufstehen. Die konditionale Analyse konnte dieser Tatsache nicht gerecht werden. Anders die Erfolgstheorie. Für den Komapatienten gilt nicht, dass er in einer hinreichend großen Anzahl von relevanten Situationen, in denen er die Intention ausbildet, aus dem Bett aufzustehen, tatsächlich aus dem Bett aufsteht. Der Grund hat mit der Einschränkung auf die relevanten Situationen zu tun. Wenn wir wissen wollen, ob der Komapatient angesichts seines Komas aufstehen kann, müssen wir das Koma über die möglichen Situationen hinweg festhalten; nur Komasituationen sind relevant. ${ }^{16}$

Als Nächstes müssen wir die relevanten Situationen laut Erfolgstheorie auf die Situationen einschränken, in denen der Patient die entsprechende Intention ausbildet. Und hier stellen wir im Komafall fest: Es gibt keine. Schließlich liegt der Patient im Koma. Es ist daher nicht der Fall, dass der Akteur in einer hinreichenden Anzahl von relevanten Situationen, in denen er intendiert aufzustehen, aus dem Bett aufsteht. Das Verhältnis der Aufstehsituationen $\mathrm{zu}$ den relevanten Intentionssituationen ist unspezifiziert. Und wenn es das Verhältnis nicht gibt, ist es a fortiori auch nicht hinreichend groß.

${ }^{15}$ Im Unterschied zur konditionalen Analyse trägt die Erfolgstheorie nicht nur der Tatsache Rechnung, dass Fähigkeiten in Graden vorliegen können; sie erlaubt auch ein Verständnis der Unterscheidung zwischen generellen und speziellen Fähigkeiten, die die konditionale Analyse vor Probleme stellt. Vgl. zu beiden Punkten Jaster (2016).

${ }^{16}$ Das heißt nicht, dass wir das Koma in jedem Kontext festhalten müssen. Wir können uns auch dafür interessieren, was eine Komapatientin angesichts derjenigen Eigenschaften tun kann, die trotz des Komas erhalten sind. Der Arzt mag etwa über die komatöse Jonglagekünstlerin sagen: „Keine Sorge. Sie kann nach wie vor jonglieren." 
Analog im Fall des Phobikers: Wenn wir wissen wollen, was der Phobiker angesichts seiner Phobie tun kann, müssen wir die Phobie über die relevanten Situationen hinweg festhalten. In keiner dieser Situationen fällt der Phobiker die Entscheidung, die Spinne zu berühren. Also gilt auch hier, dass die modale Erfolgsquote unspezifiziert und damit nicht hinreichend groß ist.

Es handelt sich hier nicht bloß um einen technischen Kniff. Vielmehr lässt sich aus der Erfolgstheorie die entscheidende zusätzliche notwendige Bedingung für das Vorliegen von Fähigkeiten ableiten, die durch Fälle verhinderter Entscheidungen zutage gefördert wurde: Um die Fähigkeit zu haben, $\varphi$ zu tun, muss es dem Akteur zunächst einmal möglich sein, sich zu entscheiden bzw. zu intendieren, $\varphi$ zu tun. Diese Bedingung ist in der Erfolgstheorie implizit enthalten, insofern die Erfolgstheorie nur dann erfüllt sein kann, wenn die Menge der relevanten Intentionssituationen nicht leer ist. Nennen wir dies die „Möglichkeitsimplikation“. Die Möglichkeitsimplikation ist im Fall des Komas und der Phobie nicht erfüllt. Die Erfolgstheorie sagt daher korrekt voraus, dass der Komapatient nicht aus dem Bett aufstehen und der Phobiker die Spinne nicht berühren kann. Damit ist die Erfolgstheorie der konditionalen Analyse um einen entscheidenden Schritt voraus. ${ }^{17}$

\section{Generelle und spezifische Fähigkeiten}

Die Überlegungen des letzten Abschnitts machen eins ganz deutlich: $\mathrm{Ob}$ jemand die Fähigkeit hat, $\varphi$ zu tun, ist keine einfache Ja-oder-Nein Angelegenheit. Vielmehr hängt es in der Regel vom Kontext ab, ob wir ihm diese Fähigkeit zuschreiben oder nicht. In unserem Zusammenhang ist die Unterscheidung von generellen und spezifischen Fähigkeiten von besonderer Bedeutung. Kann eine Person, der man alle Schläger gestohlen hat, Tennis spielen? „Das hängt davon ab“, möchte man antworten.

$[C]$ onsider a well-trained tennis player equipped with ball and racquet, standing at the service line. There is, as it were, nothing standing between him and a serve: every prerequisite for his serving has been met. Such an agent $[\ldots]$ has the specific ability to serve. In contrast, consider an otherwise similar tennis player who lacks a racquet and ball, and is miles away from a tennis court.

${ }^{17}$ Andere Eigenschaften von Fähigkeiten, die für andere Theorien problematisch sind, etwa Maskierungen, die Unterscheidung zwischen generellen und speziellen Fähigkeiten sowie Grade von Fähigkeiten erweisen sich ebenfalls als unproblematisch (vgl. Jaster 2016). 
There is clearly a good sense in which such an agent has the ability to hit a serve: he has been trained to do so, and has done so many times in the past. Yet such an agent lacks the specific ability to serve [...] Let us say that such an agent has the general ability to serve. (Maier 2014, Abschnitt 1.3)

Ähnlich Whittle:

Walter may have the ability to walk even though he is bound to a chair. Sally may have the ability to sing even though she freezes whenever her Aunt is present. Chip may have the ability to cook even though no cooking equipment is available to him now. (Whittle 2010,2)

Was diese Beispiele zeigen, ist, dass jemand durchaus die generelle Fähigkeit haben kann, $\varphi$ zu tun, auch wenn äußere oder innere Umstände ihn im Augenblick daran hindern. Die spezifische Fähigkeit, $\varphi$ zu tun, hat aber nur, wer auch in Situationen, die der gegenwärtigen bis in viele Details gleichen, hinreichend oft $\varphi$ ausführen würde, wenn er $\varphi$ intendieren würde. ${ }^{18}$ Im spezifischen Sinn kann Walter eben nicht aufstehen, wenn er festgebunden ist, Sally kann nicht singen, wenn ihre Tante zuhört, und Chip kann nicht ohne Kochutensilien kochen.

Im Rahmen der Erfolgstheorie von Fähigkeiten lässt sich die Unterscheidung zwischen generellen und spezifischen Fähigkeiten sehr gut systematisieren. Der Theorie zufolge hat ein Akteur genau dann die Fähigkeit, $\varphi$ zu tun, wenn er in einer hinreichenden Anzahl von relevanten Situationen, in denen er intendiert, $\varphi$ zu tun, auch tatsächlich $\varphi$ tut. Entscheidend ist hier der Begriff der Relevanz. Entscheidend ist, welche Situationen im jeweiligen Kontext als relevant gelten.

In Falle genereller Fähigkeiten sind wir daran interessiert zu erfahren, was eine Person tun kann, unabhängig von der aktuellen Situation, in der sie sich befindet. Wir sind interessiert an den Fähigkeiten der Person im Hinblick auf ihre dauerhaften, robusten und hauptsächlich inneren Eigenschaften. In diesem Fall umfassen die relevanten Situationen gerade die Situationen, die der aktuellen in Hinsicht auf diese Eigenschaften gleichen. Die vorübergehenden, leicht wandelbaren und zumeist äußeren Merkmale der aktuellen Situation - ob die Person einen Tennisschläger zur Hand hat, ob sie festgebunden ist, aber auch, ob ihr Bein derzeit gebrochen ist - können variieren.

Im Falle spezifischer Fähigkeiten interessiert uns dagegen, was eine Person in genau der Situation tun kann, in der sie sich befindet. Hier umfassen die relevanten Situationen deshalb nur Situationen, die der aktuellen Situation nicht nur im Hinblick auf ihre dauerhaften, robusten und hauptsächlich

Vgl. auch Vihvelin 2004, 448, Fn. 3. 
inneren Eigenschaften, sondern auch im Hinblick auf die vorübergehenden, leicht wandelbaren und zumeist äußeren Eigenschaften des Akteurs in der aktuellen Situation gleichen.

Der geübte Tennisspieler hat demnach die generelle Fähigkeit, Tennis zu spielen, auch dann, wenn er keinen Schläger zur Hand hat, weil er in einer hinreichenden Anzahl von Situationen, in denen seine dauerhaften, robusten und hauptsächlich inneren Eigenschaften konstant gehalten werden und er intendiert, Tennis zu spielen, erfolgreich Tennis spielt. Und Sally hat die generelle Fähigkeit zu singen auch dann, wenn ihre Tante anwesend ist, weil sie in einer hinreichenden Anzahl von Situationen, in denen sie ihre dauerhaften, robusten und hauptsächlich inneren Eigenschaften beibehält und intendiert, zu singen, singt. Der fehlende Tennisschläger bzw. die Tatsache, dass die Tante anwesend ist, werden im Falle genereller Fähigkeiten über die relevanten Situationen hinweg variiert.

Weder der Tennisspieler noch Sally haben in den beschriebenen Situationen aber die spezifische Fähigkeit, Tennis zu spielen bzw. zu singen. Wenn wir uns für spezifische Fähigkeiten interessieren, halten wir auch die vorübergehenden, leicht wandelbaren und oft äußeren Eigenschaften des Akteurs in der Situation fest. Wir halten also fest, dass der Tennisspieler keinen Schläger hat und dass Sallys Tante anwesend ist. Für beide gilt jetzt nicht mehr, dass sie in einer hinreichenden Anzahl der so festgelegten relevanten Situationen, in denen sie intendieren, Tennis zu spielen bzw. zu singen, Tennis spielen bzw. singen. Der Unterschied zwischen generellen und spezifischen Fähigkeiten ist demnach ein Unterschied in der Festsetzung bestimmter Relevanzkriterien für Situationen.

Es ist wichtig, sich klarzumachen, dass diese Relevanzkriterien auch innerhalb der Klasse der generellen und innerhalb der Klasse der spezifischen Fähigkeiten variieren können. Auch ein Phobiker hat die sehr generelle Fähigkeit, Spinnen zu berühren: Wenn wir von der Phobie absehen, kann er es. Dennoch halten wir in aller Regel die Phobie auch dann fest, wenn wir uns dafür interessieren, was er ganz allgemein tun kann, weil es sich um eine dauerhafte, innere Beeinträchtigung handelt. Es gibt also nicht die generelle Fähigkeit, Spinnen zu berühren. Vielmehr gibt es verschiedene mehr oder weniger generelle Fähigkeiten, Spinnen zu berühren.

Dasselbe gilt für spezifische Fähigkeiten. Auch im Spektrum der spezifischen Fähigkeiten lassen sich unterschiedliche Fähigkeiten danach unterscheiden, wie vollständig wir verschiedene Aspekte der Situation festhalten. Dieser Punkt ist im Zusammenhang mit der Frage nach freiheitsrelevanten Fähigkeiten von entscheidender Bedeutung. 
Nennen wir Fähigkeiten, bei denen ausnahmslos alle Aspekte der aktuellen Situation relevant sind, partikulare Fähigkeiten. Jemand besitzt demnach die partikulare Fähigkeit, $\varphi$ zu tun, wenn er in einer hinreichenden Anzahl der Situationen, die der aktuellen Situation in allen Aspekten gleichen und in denen er die Intention ausbildet, $\varphi$ zu tun, auch tatsächlich $\varphi$ tut. Mit anderen Worten: Bei partikularen Fähigkeiten schrumpft die Menge der relevanten Situationen zusammen auf nur eine - die aktuale Situation. Und damit wird die Bedingung ,in einer hinreichenden Anzahl“ zu einer Alles-oder-Nichts Angelegenheit.

Wenn eine Person in einer Situation intendiert, $\varphi$ zu tun, und damit Erfolg hat, besitzt sie in dieser Situation die partikulare Fähigkeit, $\varphi$ zu tun. Wenn sie in dieser Situation intendiert, $\varphi$ zu tun, und scheitert, besitzt sie in dieser Situation die partikulare Fähigkeit, $\varphi$ zu tun, nicht. Und wie ist es, wenn die Person in der gegebenen Situation die Intention, $\varphi$ zu tun, gar nicht ausbilden kann - z.B. weil determiniert ist, dass sie in dieser Situation nicht intendiert, $\varphi$ zu tun? Auch dann besitzt die Person der Erfolgstheorie von Fähigkeiten zufolge die entsprechende partikulare Fähigkeit nicht. Der Fall ist dann analog zum Komafall - die Menge der relevanten Intentionssituationen ist leer. ${ }^{19}$

Partikulare Fähigkeiten sind offensichtlich eine extreme Form spezifischer Fähigkeiten. Für die relevanten Situationen werden wirklich alle Merkmale der aktuellen Situation festgehalten. Aber das muss nicht sein; es gibt auch weniger extreme Formen spezifischer Fähigkeiten. Denkbar ist etwa, dass wir bei der Frage, was ein Akteur in einer bestimmten Situation tun kann, einige wenige, häufig mentale Merkmale außer Acht lassen.

Wir können z.B. bei der Frage, ob eine Person in einer bestimmten Situation die Fähigkeit hat zu schwimmen, davon absehen, dass die Person in dieser Situation eine große Abneigung gegen das Schwimmen hat. Diese Person hat sicher nicht die partikulare Fähigkeit zu schwimmen. Aber sie kann, in einem etwas weniger extremen Sinn, dennoch die entsprechende spezifische Fähigkeit besitzen. Wenn wir ihre Abneigung außer Acht und somit offenlassen, ob die Person Lust zum Schwimmen hat oder nicht, kann es durchaus sein, dass sie in hinreichend vielen Situationen, die der aktuellen bis auf den genannten Punkt gleichen und in denen sie die Intention ausbildet zu schwimmen, auch tatsächlich schwimmt. Sagen wir, dass die Person in diesem Fall die konative Fähigkeit hat zu schwimmen. ${ }^{20}$

19 Vgl. oben S. 172f.

${ }^{20}$ Wir folgen hier Berofsky (2002), der von ,conative abilities“ spricht. 
Handelt es sich bei konativen Fähigkeiten tatsächlich um spezifische Fähigkeiten? Ja, denn wir fragen immer noch danach, was eine Person in einer spezifischen Situation tun kann. Spezifische Fähigkeiten können also im Hinblick auf die Vollständigkeit der Merkmale der aktuellen Situation variieren, die für die entsprechenden relevanten Situationen relevant sind. Wir können von einem kleinen Teil dieser Merkmale abstrahieren und trotzdem davon reden, was die Person in genau der Situation, in der sie sich befindet, tun kann. Tatsächlich abstrahieren wir sogar normalerweise von den Umständen, die die motivationalen Zustände der Person determinieren. Berofsky drückt das so aus:

[A] person who has the power to act may fail to do so for reasons having to do with her motivation or her will. [...] We normally distinguish between these [conative conditions] and the other conditions (ability, opportunity), regarding only the latter's absence as depriving one of the power to act. (Berofsky 2002, 196)

Berofsky betont hier, dass wir normalerweise nicht sagen, dass eine Person in einer bestimmten Situation unfähig ist, $\varphi$ zu tun, nur weil sie in dieser Situation eine Abneigung dagegen hat, $\varphi$ zu tun. Spezifische Fähigkeiten, bei denen wir von den motivationalen Zuständen einer Person abstrahieren, bilden also eine wichtige Klasse spezifischer Fähigkeiten.

Partikulare und konative Fähigkeiten sind nur zwei Beispiele für eine Vielzahl unterschiedlicher Zuschnitte spezifischer Fähigkeiten. Wie umfassend wir die Tatsachen der aktualen Situation festhalten und welche Details wir variieren, kann sich auf vielfältige Weise unterscheiden. Im Zusammenhang mit der Frage nach den freiheitsrelevanten Fähigkeiten sind jedoch partikulare und konative Fähigkeiten zwei relevante Zuschnitte, weil sie dabei helfen zu sehen, dass die Antwort auf die Frage komplexer ist, als gemeinhin angenommen.

\section{Determinismus und freiheitsrelevante Fähigkeiten}

Kommen wir zurück zur Ausgangsfrage. Kann man in einer Situation die Fähigkeit besitzen, $\varphi$ zu tun, wenn in dieser Situation determiniert ist, dass man nicht $\varphi$ tut?

Was die Diskussion der letzten Abschnitte gezeigt haben dürfte, ist, dass man hier offenbar differenzieren muss. Wenn in einer konkreten Situation unter anderem durch die Tatsache, dass Sallys Tante anwesend ist - determiniert ist, dass Sally nicht singt, selbst wenn sie das intendiert, scheint es trotzdem durchaus möglich, dass sie in dieser Situation eine entsprechende generelle Fähigkeit besitzt. Wenn wir die Anwesenheit von Sallys Tante 
über die möglichen Situationen hinweg variieren, spricht offenbar nichts dagegen, dass Sally in hinreichend vielen dieser Situationen, in denen sie intendiert zu singen, erfolgreich singt.

Aber wie ist es mit spezifischen Fähigkeiten? An dieser Stelle wird die Einsicht relevant, dass es die spezifische Fähigkeit, $\varphi$ zu tun, gar nicht gibt. Vielmehr lassen sich auch im Spektrum der spezifischen Fähigkeiten unterschiedliche Fähigkeiten danach unterscheiden, wie vollständig wir verschiedene Aspekte der Situation festhalten.

Fragen wir also zuerst: Kann ein Akteur die partikulare Fähigkeit haben, $\varphi$ zu tun, wenn determiniert ist, dass er nicht $\varphi$ tut? Offenbar nicht. Denn es gibt nur zwei Möglichkeiten: Entweder es ist determiniert, dass er $\varphi$ nicht intendiert. Den Fall haben wir uns gerade angeschaut. Oder es ist determiniert, dass er $\varphi$ intendiert und dass er $\varphi$ trotzdem nicht ausführt; dann fehlt ihm die partikulare Fähigkeit, $\varphi$ zu tun, weil die modale Erfolgsquote nicht hoch genug ist.

Anders verhält es sich beispielsweise mit konativen Fähigkeiten. Denn genau wie generelle Fähigkeiten sind auch diese Fähigkeiten offenbar mit dem Determinismus vereinbar. $\mathrm{Zu}$ den Faktoren, die determinieren, was eine Person in einer bestimmten Situation tut, gehören in aller Regel gerade auch ihre motivationalen Zustände. Wenn wir von diesen abstrahieren, ist es daher durchaus möglich, dass die Person in einer hinreichenden Anzahl der relevanten Situationen, in denen sie intendiert, $\varphi$ zu tun, auch tatsächlich $\varphi$ ausführt.

Allgemein lässt sich also Folgendes festhalten. Für die Frage, ob eine Person, die in einer aktuellen Situation nicht $\varphi$ tut, in dieser Situation trotzdem die Fähigkeit hat, $\varphi$ zu tun, ist entscheidend, welches die für die Fähigkeit relevanten möglichen Situationen sind. Und deshalb gilt: Wenn der Determinismus wahr ist, hat die Person die Fähigkeit zu pen nicht, falls bei den für diese Fähigkeit relevanten möglichen Situationen (neben den Naturgesetzen) alle Bedingungen der aktuellen Situation festgehalten werden, die kausal hinreichend dafür sind, dass die Person $\varphi$ nicht ausführt. Denn wenn das so ist, gibt es keine relevante mögliche Situation, in der die Person intendiert, $\varphi$ zu tun, und dann auch wirklich $\varphi$ tut. Für partikulare Fähigkeiten trifft das zu, für generelle Fähigkeiten nicht. Und bei den spezifischen Fähigkeiten gibt es zumindest einige, z.B. die konativen Fähigkeiten, bei denen die relevanten möglichen Situationen nicht alle Bedingungen der aktuellen Situation umfassen, die kausal hinreichend dafür sind, dass die Person $\varphi$ nicht ausführt.

Die Grundidee fähigkeitsbasierter Freiheitstheorien ist, dass wir genau dann frei sind, wenn wir über bestimmte Fähigkeiten verfügen. Keil hat 
bezweifelt, dass wir diese Fähigkeiten auch dann besitzen können, wenn der Determinismus wahr ist. In den letzten Abschnitten sollte aber eins klar geworden sein: Mit Blick auf die Vereinbarkeit von Freiheit und Determinismus ist entscheidend, zu welcher Art von Fähigkeiten die für Freiheit konstitutiven Fähigkeiten gehören.

Wir denken, dass dabei eins klar ist: Bei den für Freiheit entscheidenden Fähigkeiten geht es nicht um generelle Fähigkeiten. Wenn ein Akteur ein gebrochenes Bein hat, behält er die generelle Fähigkeit zu schwimmen. Aber wenn wir uns fragen, ob er ein ertrinkendes Kind hätte retten können, fragen wir ganz offensichtlich nicht nach dieser generellen Fähigkeit. Dass sein Bein gebrochen ist, sorgt dafür, dass der Akteur das Kind im relevanten Sinne nicht hätte retten können.

Uns scheint, es geht auch nicht um partikulare Fähigkeiten. Partikulare Fähigkeiten bilden den Extremfall unter mehr oder weniger spezifischen Fähigkeiten, weil sie keinen modalen Charakter aufweisen. Die Frage, was wir in einer konkreten Situation hätten tun können, ist in aller Regel noch nicht beantwortet, wenn wir wissen, was wir in dieser Situation tatsächlich getan haben. Verstehen wir „können“ aber im Sinne partikularer Fähigkeiten, dann fallen diese Fragen zusammen. Man kann dann immer nur genau das tun, was man tatsächlich tut. Laut der Erfolgstheorie haben wir es hier mit einem legitimen Sinn von „,können“ zu tun: angesichts der Vollständigkeit der Tatsachen kann stets jeder nur das tun, was er tatsächlich tut. Aber es ist ein Sinn von „können“, der wenig mit unseren üblichen Verwendungen des Ausdrucks zu tun hat. In aller Regel sind Könnenszuschreibungen modal in dem Sinne, dass "S kann $\varphi$ tun“ wahr sein kann, auch wenn S nicht tatsächlich $\varphi$ tut. Ein Sinn von „können“, der dieses Merkmal nicht aufweist, hat wenig mit Könnensaussagen im herkömmlichen Sinne zu tun. Es ist daher nicht plausibel, dass dieser Sinn von „,können“ die Fähigkeiten einschließt, die für Freiheit und Verantwortung relevant sind.

Vor dem Hintergrund der letzten Abschnitte stellt sich der Disput zwischen Kompatibilisten und Inkompatibilisten also folgendermaßen dar: Fähigkeitsinkompatibilisten sind der Meinung, dass es sich bei den für Freiheit relevanten Fähigkeiten um sehr spezifische Fähigkeiten handelt. Hinter dieser Auffassung steht offenbar folgende Idee. Nehmen wir dieses Mal als Beispiel Keils Fähigkeit, weiter zu überlegen. Eine Person kommt in eine Situation, in der sie die Wahl zwischen zwei Handlungen A und B hat. Sie überlegt, wägt die Gründe ab und kommt dabei zu dem Ergebnis, dass die Gründe für A deutlich überwiegen. Das beendet den Prozess des Überlegens zum Zeitpunkt t. Die Person bildet die Intention aus, A zu tun, und tut dann auch A. 
Der Determinist sagt, dass es sich bei dem ganzen Prozess des Überlegens, des Ausbildens der Intention und der Ausführung der Handlung um einen deterministischen Prozess handelt. Zu jedem Zeitpunkt war durch vorhergehende Ereignisse determiniert, was als Nächstes passiert. Und so war auch determiniert, dass die Person ihre Überlegung zum Zeitpunkt $\mathrm{t}$ beendet. Wie hätte sie also zu diesem Zeitpunkt weiter überlegen können?

Das mag prima facie plausibel klingen. Aber offensichtlich ist diese Überlegung nur überzeugend, wenn man davon ausgeht, dass bei den für die Fähigkeit weiter zu überlegen relevanten möglichen Situationen alle Umstände festgehalten werden müssen, die dafür kausal hinreichend waren, dass die Person nicht weiter überlegte.

Fähigkeitskompatibilisten dagegen sind offenbar der Meinung, dass es sich bei den freiheitsrelevanten Fähigkeiten keineswegs um besonders spezifische Fähigkeiten handelt, dass bei den für diese Fähigkeiten relevanten möglichen Situationen vielmehr auch Umstände variiert werden dürfen, die zu den Umständen gehören, die kausal dafür hinreichend waren, dass der Handelnde $\varphi$ nicht ausführte.

Mit dieser Auffassung können sie sich unter anderem auf die von Berofsky angeführten Fälle konativer Fähigkeiten stützen, in denen wir aus der Tatsache, dass jemand in einer bestimmten Situation nicht motiviert ist, $\varphi$ zu tun, oder dass er eine Abneigung dagegen hat, $\varphi$ zu tun, nicht schließen, dass er in dieser Situation $\varphi$ nicht tun kann. So sagen wir von einem Schwimmer, dem das Wasser zu kalt ist, nicht, dass er nicht schwimmen kann, sondern dass er nicht schwimmen will. Und ähnlich sehen wir die Dinge in anderen Fällen, in denen die Motive einer Person sie hindern, eine bestimmte Handlung auszuführen. Der Vegetarier könnte Fleisch essen; aber er will es nicht. Die moralische Person hätte die Brieftasche stehlen können; aber ihr Wunsch, moralisch korrekt zu handeln, hielt sie davon ab. Hans hätte um Hilfe bitten können, wollte sich aber keine Blöße geben.

Wenn wir fragen, was jemand in einer spezifischen Situation hätte tun können oder ob er in dieser Situation etwas anderes hätte tun können, abstrahieren wir also häufig von den Motiven des Akteurs. Und da Aussagen wie diese im Alltag sehr verbreitet sind, spricht prima facie viel dafür, dass es sich bei den für Freiheit entscheidenden Fähigkeiten keineswegs um so spezifische Fähigkeiten handelt, wie Fähigkeitsinkompatibilisten meinen. Die Motive eines Handelnden gehören nämlich sicher zu den Umständen, die kausal dafür hinreichend waren, dass der Handelnde $\varphi$ nicht ausführte. Wenn wir diese Motive variieren dürfen, können wir deshalb durchaus annehmen, dass eine Person, die (unter anderem aufgrund ihrer aktuellen Motive) in einer Situation determiniert ist, nicht zu schwimmen, trotzdem über 
die Fähigkeit verfügt zu schwimmen, da es dann ja zumindest möglich ist, dass es hinreichend viele relevante mögliche Situationen gibt, in denen die Person (aufgrund anderer Motive) intendiert $\mathrm{zu}$ schwimmen und schwimmt.

\section{Ist die Debatte damit beendet?}

Nach dieser Diagnose könnte man denken, dass im Disput zwischen Fähigkeitsinkompatibilisten und Fähigkeitskompatibilisten ein argumentatives Patt besteht. Fähigkeitsinkompatibilisten gehen davon aus, dass bei den für freiheitskonstitutive Fähigkeiten relevanten möglichen Situationen alle Umstände festgehalten werden müssen, die kausal dafür hinreichend waren, was die Person tatsächlich intendiert und was sie tatsächlich getan hat. Fähigkeitskompatibilisten dagegen meinen, dass bei den für freiheitskonstitutive Fähigkeiten relevanten möglichen Situationen auch diese Umstände variiert werden dürfen.

Das könnte man auch so ausdrücken: Fähigkeitsinkompatibilisten und Fähigkeitskompatibilisten haben unterschiedliche Auffassungen hinsichtlich der Bedingungen, unter denen man eine Entscheidung oder Handlung wirklich frei nennen darf, und reden insofern aneinander vorbei. Die Fähigkeiten, die die jeweiligen Lager für freiheitsrelevant halten, wären in diesem Fall einfach nicht dieselben.

Wir neigen allerdings zu der Auffassung, dass sich zu dem Dissens zwischen Fähigkeitsinkompatibilisten und Fähigkeitskompatibilisten noch mehr sagen lässt.

Nehmen wir als Beispiel einen Phobiker, sagen wir einen Spinnenphobiker, der insofern nicht frei ist, als er nicht in der Lage ist, eine Spinne anzufassen, während ein gesunder Erwachsener das durchaus kann. Worin besteht der Unterschied zwischen den beiden Personen? Ist der Spinnenphobiker nicht frei, weil er determiniert ist, die vor ihm liegende Spinne nicht anzufassen, während bei dem gesunden Erwachsenen keinerlei diesbezügliche Determination vorliegt?

Nach der Lockeschen Auffassung ist das zumindest nicht die ganze Wahrheit. Unfrei ist der Spinnenphobiker nach Locke, weil ihm eine der beiden Fähigkeiten fehlt: 1. Die Fähigkeit zu überlegen, ob es besser wäre, die Spinne anzufassen oder nicht. 2. Die Fähigkeit, dem Ergebnis dieser Überlegung gemäß zu handeln. ${ }^{21}$ Wir können annehmen, dass der Spinnen-

21 Hier zeigt sich im Übrigen, dass Keils Freiheitstheorie wohl doch keine gute Alternative zur Theorie Lockes ist. Denn nach Keil hängt Freiheit von der Fä- 
phobiker über die erste der beiden Fähigkeiten verfügt. Seine Unfreiheit beruht darauf, dass ihm die zweite Fähigkeit fehlt.

Woran liegt das? Daran dass er selbst dann, wenn er beim Überlegen zu dem Ergebnis kommt, dass es besser wäre, die Spinne anzufassen, und wenn er sich auch wünscht, dem Ergebnis seiner Überlegung gemäß zu handeln, aufgrund seiner Phobie trotzdem die Intention ausbildet, die Spinne nicht anzufassen. Wenn wir versuchen, eine Antwort auf die Frage zu finden, ob der Spinnenphobiker über Lockes zweite Fähigkeit verfügt, halten wir also die Spinnenphobie fest, fragen uns aber, was - bei gegebener Phobie - passiert wäre, wenn der Spinnenphobiker überlegt hätte, wenn er bei dieser Überlegung zu dem Ergebnis gekommen wäre, dass es besser wäre, die Spinne anzufassen, und wenn er außerdem den Wunsch gehabt hätte, diesem Ergebnis seiner Überlegung gemäß zu handeln. Wir variieren also auch Tatsachen, die das Überlegen des Spinnenphobikers betreffen.

Auch wenn er nicht überlegt hat und nach Voraussetzung determiniert war, nicht zu überlegen, fragen wir uns, was passiert wäre, wenn er überlegt hätte. Und insbesondere fragen wir uns, was passiert wäre, wenn diese Überlegung zu dem Ergebnis geführt hätte, dass es besser wäre, die Spinne anzufassen. Wir halten den Spinnenphobiker für unfrei, weil wir nach allem, was wir über Phobien wissen, davon ausgehen, dass der Spinnenphobiker auch dann die Spinne nicht angefasst hätte. Bei der Beantwortung der Frage, ob der Spinnenphobiker über die zweite der Lockeschen Fähigkeiten verfügte, variieren wir also auch Umstände, die zu den kausal hinreichenden Umständen dafür gehören, dass der Spinnenphobiker die Spinne nicht anfasste. Entscheidend ist nicht, ob er determiniert war, die Spinne nicht anzufassen, sondern welchen Einfluss die Spinnenphobie auf seine Entscheidung und sein Handeln hatte. Und genau um das herauszufinden, halten wir die Spinnenphobie fest, während viele andere Umstände der aktuellen Situation variiert werden.

Wie ist es nun mit einem gesunden Erwachsenen, Paul, der nicht unter Phobien oder ähnlichen Störungen leidet? Paul wird gebeten, eine Spinne anzufassen, die vor ihm auf dem Tisch herumkrabbelt; aber er tut es nicht z.B. weil er dringend seinen Bruder anrufen möchte und deshalb aufsteht und das Zimmer verlässt. Nehmen wir an, dass der Prozess, der zu der Ent-

higkeit ab, weiter zu überlegen. Aber ist es wirklich plausibel anzunehmen, dass der Spinnenphobiker deshalb nicht frei ist, weil er nicht über die Fähigkeit verfügt, weiter zu überlegen? Selbst wenn er über diese Fähigkeit verfügte, würde das doch nichts ändern. Entscheidend ist wirklich, dass er nicht in der Lage ist, dem Ergebnis seiner Überlegung gemäß zu handeln. 
scheidung geführt hat, die Spinne nicht anzufassen, sondern aufzustehen und rauszugehen, ein deterministischer Prozess war. Würde das bedeuten, dass Paul in seiner Entscheidung nicht frei war?

Noch einmal: Locke zufolge wäre Paul nicht frei, wenn er nicht hätte innehalten und überlegen können oder wenn er nicht dem Ergebnis dieser Überlegung gemäß hätte handeln können. Angenommen, der Prozess, der zu Pauls Entscheidung geführt hat, war ein deterministischer Prozess. Würde das bedeuten, dass er über mindestens eine dieser Fähigkeiten nicht verfügt? Welche Umstände müssen wir berücksichtigen, wenn wir eine Antwort auf diese Frage suchen?

Wir denken, dass es nicht angemessen ist, alle Umstände festzuhalten, die in der aktuellen Situation dafür kausal hinreichend waren, dass Paul die Spinne nicht angefasst hat, sondern aufgestanden und hinausgegangen ist. Vielmehr ist auch relevant, was in der folgenden, nicht gänzlich gleichen Situation passiert wäre: In dem Moment, in dem sich Paul anschickt, aufzustehen und den Raum zu verlassen, sagt eine Person: „Paul, wenn Du jetzt den Raum verlässt, ruinierst Du die ganze Versuchsanordnung.“

Mag sein, dass Pauls Wunsch, seinen Bruder anzurufen, zu groß ist, dass er trotzdem den Raum verlässt. Mag aber auch sein, dass Paul sich sagt: Ok, so wichtig ist das jetzt doch nicht; ich kann auch noch einen Moment warten und erst noch das Experiment zu Ende bringen. Und dann kommt es erstens darauf an, wie oft Paul, wenn er mit neuen Argumenten konfrontiert wird, tatsächlich beginnt zu überlegen. Wenn das häufig genug passiert, hat er offenbar die Fähigkeit, vor dem Handeln innezuhalten und zu überlegen. Und es kommt zweitens darauf an, wie oft er, wenn er bei diesen Überlegungen zu dem Ergebnis kommt, dass es das Beste wäre, die Spinne anzufassen, dies auch tatsächlich tut. Dann hat er auch die Fähigkeit, dem Ergebnis seiner Überlegung gemäß zu handeln.

Wir denken deshalb, dass die Lockeschen Fähigkeiten zwar spezifische, aber keine so spezifischen Fähigkeiten sind, wie Fähigkeitsinkompatibilisten meinen, da wir uns z.B. immer auch fragen müssen, was passiert wäre, wenn Paul mit neuen Argumenten konfrontiert worden wäre.

Damit wird auch klar, dass ein letztes Argument des Fähigkeitsinkompatibilisten ebenfalls nicht sticht. Der Fähigkeitsinkompatibilist könnte argumentieren, die Überlegungen des Abschnitts 2 hätten doch gezeigt, dass eine Person nicht über die Fähigkeit verfügt, $\varphi$ zu tun, wenn es ihr in der betreffenden Situation nicht möglich ist, die Intention, $\varphi$ zu tun, überhaupt auszubilden. Aber wenn determiniert ist, dass sie die Intention, $\varphi$ zu tun, nicht ausbildet, dann kann sie diese Intention offenbar nicht ausbilden. Also hat sie in diesem Fall auch nicht die die Fähigkeit, $\varphi$ zu tun. 
Doch auch hier ist die Frage, wie das „kann“ in der Aussage „S kann die Intention, $\varphi$ zu tun, nicht ausbilden“" zu verstehen ist. Der Komapatient wird die Intention aufzustehen nicht ausbilden - in allen Situationen, in denen er im Koma liegt. Der Phobiker wird die Intention, die Spinne anzufassen, nicht ausbilden - in allen Situationen, in denen seine Phobie vorliegt. Aber welche Situationen müssen wir in Betracht ziehen, wenn es darum geht, ob ein gesunder Erwachsener wie Paul intendieren kann, die Spinne anzufassen? Der Fähigkeitsinkompatibilist wird sagen: Nur Situationen, in denen alle Umstände realisiert sind, die für das aktuelle Verhalten Pauls kausal hinreichend waren. Und weil offenbar in all diesen Situationen determiniert ist, dass Paul nicht intendiert, die Spinne anzufassen, hat Paul in der gegebenen Situation auch nicht die Fähigkeit, die Spinne anzufassen. Aber wenn er so argumentiert, begeht der Fähigkeitsinkompatibilist ganz offensichtlich eine petitio principii; denn es geht ja gerade um die Frage, wie das ,kann“ zu verstehen ist.

Tatsächlich entspricht die Position des Fähigkeitsinkompatibilisten aber auch nicht unserem alltäglichen Vorgehen, wenn wir wissen wollen, was Paul in der gegebenen Situation intendieren kann und was nicht. Wenn wir uns fragen, ob Paul in der geschilderten Situation intendieren kann, die Spinne anzufassen, sehen wir in aller Regel, ganz so wie es Berofsky beschreibt, von seiner spezifischen Motivationslage ab, in der der Wunsch, den Bruder anzurufen, stärker als andere Wünsche ist. Stattdessen fragen wir z.B., was wohl passiert wäre, wenn man Paul mit neuen Argumenten konfrontiert hätte. Wenn man einen Spinnenphobiker mit neuen Argumenten konfrontiert, ändert sich in aller Regel nichts - er wird die Intention, die Spinne anzufassen, nicht ausbilden. ${ }^{22}$ Anders bei Paul. Dass es sich bei Paul um einen gesunden Erwachsenen handelt, zeigt sich gerade daran, dass er im Allgemeinen durch (neue) Argumente dazu gebracht werden kann, andere Intentionen auszubilden.

Unser Hauptargument gegen den Fähigkeitsinkompatibilisten besteht demnach in dem Vorwurf, dass er den Unterschied zwischen normalen gesunden Erwachsenen und psychisch Kranken wie etwa Phobikern nicht erklären kann. Denn dieser Unterschied besteht nicht darin, dass der Phobiker determiniert ist, bestimmte Dinge zu tun oder nicht zu tun, während der gesunde Erwachsene nicht in diesem Sinne determiniert ist. Das zeigt sich

${ }^{22}$ Im Anschluss an andere Autoren hat z.B. Julius Schälike (2010, 77ff.) wohl zu Recht argumentiert, auch der Spinnenphobiker würde wohl die Spinne anfassen, wenn man ihm mit dem sofortigen Tod am Galgen droht, falls er es nicht tut. 
besonders klar an Fällen, in denen es für den Handelnden offensichtlich ist, was zu tun ist. Martine Nida-Rümelin nennt ihrem Aufsatz „Freedom and the Phenomenology of Agency" unter anderem folgende Beispiele:

Example 1 While Anton is sitting on a river beach he observes a 2 years old boy who is playing next to the river. The parents' attention is caught by a conversation with friends. There are no other people on the beach. Suddenly the boy stumbles and falls into the water. Anton immediately understands the situation. He jumps up, hurries to the river and saves the little boy grasping his arm and pulling him out.

Example 2 Beatrice is a teacher of mathematics. Every Monday morning Beatrice stands up at 8.00 and leaves her home to go to the school in her neighborhood in order to start teaching at 9.00. This is what she does on Monday, April 11, 2016. She doesn't think about whether or not she will go to school today. It is obvious to her as she wakes up that this is the thing to do. She realizes her plan without any deliberation. (Nida-Rümelin 2017, 15)

Es ist durchaus plausibel anzunehmen, dass Anton kausal determiniert ist, zum Fluss zu rennen und das Kind zu retten, und dass Beatrice ebenso determiniert ist, am 11. April $2016 \mathrm{um} 8.00 \mathrm{Uhr}$ aufzustehen und zu 9.00 Uhr in die Schule zu gehen, um zu unterrichten.

Trotzdem spricht nichts dagegen, dass sowohl Anton als auch Beatrice in ihrem Handeln frei und verantwortlich sind. Denn nichts spricht dagegen, dass beide über die Lockeschen Fähigkeiten verfügen. Dies wäre jedoch nicht der Fall, wenn diese Fähigkeiten so spezifische Fähigkeiten wären, wie sie Fähigkeitsinkompatibilisten im Sinn haben. Dass Anton und Beatrice über die Lockeschen Fähigkeiten verfügen, wird erst klar, wenn bei den für diese Fähigkeiten relevanten möglichen Situationen auch Bedingungen variiert werden, die zu den Umständen gehören, die für das tatsächliche Verhalten von Anton und Beatrice kausal hinreichend waren.

\section{Literatur}

Beckermann, Ansgar (2016) „Die Perspektive des Richters“, in: W. Freitag, H. Rott, H. Sturm \& A. Zinke (Hg.) Von Rang und Namen. Münster, S. 1-14. (In diesem Band S. 149-162)

Berofsky, Bernard (2002) „Ifs, cans, and free will: The issues“, in: R. H. Kane (Hg.), The Oxford Handbook of Free Will, Oxford, S. 181-201.

Chisholm, Roderick M. (1966) „Freedom and Action“, in: K. Lehrer (Hg.), Freedom and Determinism. New York, S. 11-44.

Chisholm, Roderick M. (1976) Person and Object: A Metaphysical Study, La Salle, IL. 
Hoefer, Carl (2016) „Causal Determinism“, in: The Stanford Encyclopedia of Philosophy (Spring 2016 Edition), Edward N. Zalta (ed.), URL $=<$ https://plato.stanford.edu/archives/spr2016/entries/determinism-causal/>.

Greco, John (2009) „Knowledge and Success From Ability“, in: Philosophical Studies 142, S. 17-26.

Jaster, Romy (2016) Agents’ Abilities. Dissertationsschrift. Humboldt-Universität zu Berlin. (Inzwischen 2020 als Buch bei de Gruyter erschienen)

Keil, Geert (2009) „Wir können auch anders. Skizze einer libertarischen Konzeption von Willensfreiheit", in: Erwägen Wissen Ethik 20 (1), S. 3-16.

Keil, Geert (2013) Willensfreiheit. 2. Auflage. Berlin/Boston.

Kratzer, Angelika (1977) „What ,Must' and ,Can' Must and Can Mean“, in: Linguistics and Philosophy 1, S. 337-355.

Kratzer, Angelika (1981) „The Notional Category of Modality“, in: H. J. Eikmeyer \& H. Rieser (Hg.), Words, Worlds, and Contexts: New Approaches in Word Semantics. Berlin, S. 38-74.

Lehrer, Keith (1968) „Cans without Ifs“, in: Analysis 29, S. 29-32.

Lewis, David (1976) „The Paradoxes of Time Travel“, in: American Philosophical Quarterly 13, S. 145-52.

Locke, John (1975) An Essay concerning Human Understanding. Ed. by Peter H. Nidditch. Oxford.

Maier, John (2013) „The Agentive Modalities“, in: Philosophy and Phenomenological Research 87, S. 1-22.

Maier, John (2014) „Abilities“, in: The Stanford Encyclopedia of Philosophy (Fall 2014 Edition), Edward N. Zalta (ed.), URL $=<$ http://plato.stanford.edu/ archives/fall2014/entries/abilities/>.

Manley, David \& Ryan Wasserman (2008) „On Linking Dispositions and Conditionals“, in: Mind 117, S. 59-84.

Moore, George Edward (1966) Ethics, Oxford.

Nida-Rümelin, Martine (2017) „Freedom and the Phenomenology of Agency“, in: Erkenntnis (doi:10.1007/s10670-016-9872-0)

Schälike, Julius (2010) Spielräume und Spuren des Willens. Eine Theorie der Freiheit und der moralischen Verantwortung. Paderborn.

Van Inwagen, Peter (1983) An Essay on Free Will. Oxford.

Vetter, Barbara (2015) Potentiality: From Dispositions to Modality. Oxford.

Vihvelin, Kadri (2004) „Free Will Demystified: A Dispositionalist Account“, in: Philosophical Topics 32, S. 427-450.

Vihvelin, Kadri (2013) Causes, Laws, and Free Will: Why Determinism Doesn't Matter. Oxford. 